\title{
Scenario for equilibrium solid-stabilized emulsions
}

\author{
Willem K. Kegel* and Jan Groenewold \\ Van't Hoff Laboratory for Physical and Colloid Chemistry, Debye Institute, Utrecht University, Padualaan 8, 3584 CH Utrecht, \\ The Netherlands
}

(Received 16 February 2009; revised manuscript received 4 August 2009; published 8 September 2009)

\begin{abstract}
We show theoretically that under certain conditions colloidal particles can give rise to spontaneous emulsification of oil/water systems. The capillary penalty to create a large interface is compensated by entropic contributions connected to ionic dissociation on the colloid surfaces. The colloids themselves are absorbed on the oil/water interface. The conditions for spontaneous emulsification are: (1) oil-water interfacial tension is low (a few $\mathrm{mN} / \mathrm{m}$ or lower); (2) interfacial tension between colloids and oil is smaller than between colloids and water (in the absence of charge effects); (3) density of chargeable groups on the colloids is large (order $1 \mathrm{~nm}^{-2}$ ); (4) Debye length is comparable to colloid size.
\end{abstract}

DOI: 10.1103/PhysRevE.80.030401

PACS number(s): 82.70.Kj, 41.20.Cv, 68.05.-n, 89.75.Fb

Particles in between nanometers and micrometers in size may adsorb onto oil-water interfaces, thereby stabilizing emulsions against coarsening [1,2]; see also [3,4] for reviews. These solid-stabilized-or Pickering emulsionsusually are not thermodynamically stable. Recently experimental evidence was presented indicating an exception to this rule, [5]. There, it was found that mixtures of a particular kind of oil (methacryloxypropyltrimethoxysilane, TPM), water, and magnetite colloids of 5-10 nm in size, spontaneously emulsify forming oil drops in water with the colloids adsorbed onto the oil-water interface. These drops can be in between 10 and $150 \mathrm{~nm}$ in radius and are rather monodisperse. While TPM is known to hydrolyze and form surfaceactive molecules, colloids are required for stability of the emulsion. These colloids should be charged [6], and smaller than approximately $200 \mathrm{~nm}$ [7]. Moreover, oil-water interfacial tension is low, i.e., around $2 \mathrm{mN} / \mathrm{m}[8,9]$, yet it still is two orders of magnitude higher than it is in microemulsions, i.e., thermodynamically stable oil-water-surfactant mixtures [10]. Here, we present a scenario in which the driving force for emulsification is an entropic contribution connected to ionic dissociation of chargeable groups on the colloids. The competing (free) energies are formation of an extended colloid loaded oil-water interface (unfavorable contribution) and charging and release of counterions (favorable contribution) upon adsorption from the oil phase. We will first outline the relevant contributions to the free energy of solidstabilized emulsions. Subsequently, we define the reference states to which this free energy should be compared to in order to establish if the emulsified state is thermodynamically stable. Next we calculate the electrostatic contribution, and find conditions where the emulsified state is stable with respect to the demixed state of oil and water with the colloids dispersed into one of the liquids.

Consider $n$ charged spherical particles adsorbed at a curved oil-water interface as sketched in Fig. 1. The relevant free energy of the drop per colloid in Fig. 1, where we ignore explicit line tension effects for the moment, reads as

\footnotetext{
*w.k.kegel@uu.nl
}

$$
F / n=\gamma_{c w} A_{c w}+\gamma_{c o} A_{c o}+\gamma_{o w} A_{o w}-\Delta p v_{d}+f_{e l}(\theta, \omega, a) .
$$

The interfacial tension between colloids and oil is $\gamma_{c o}$, and between colloids and water it is $\gamma_{c w}$. This last value is the interfacial tension where electrostatic effects are absent. The interfacial tension between oil and water is $\gamma_{o w}$. The radius of the assumed spherical oil-water meniscus is $R_{m}$, and the radius defined as the distance between the drop center and the particle center is $R_{0}$. The area of the colloid-water interface per adsorbed colloid in Eq. (1) is $A_{c w}=2 \pi a^{2}[1+\cos (\theta-\omega)]=2 \pi a^{2}\left(1+\frac{R_{0}-h}{a}\right)$, that of the colloid-oil interface is $A_{c o}=2 \pi a^{2}[1-\cos (\theta-\omega)]$ $=2 \pi a^{2}\left(1-\frac{R_{0}-h}{a}\right)$, and the total area of the bare oil-water interface $n A_{o w}=4 \pi R_{m}^{2}-2 \pi R_{m}^{2} n(1-\cos \omega)$ $=4 \pi R_{m}^{2}-2 \pi R_{m}^{2} n\left(1-\frac{h}{R_{m}}\right)$, where $h=R_{m}-\frac{\left(a-R_{m}+R_{0}\right)\left(a+R_{m}-R_{0}\right)}{2 R_{0}}$ is the distance between the center of the (flat) circle defined by the three-phase (oil, water, colloid) line and the drop center. $n v_{d}$ is the volume of the liquid part of the drop in Fig. 1. $\Delta p$ is the difference between the pressure inside and outside the drop. The contact or wetting angle, $\theta$, and apex angle between contact line, drop center and particle center, $\omega$, are indicated in Fig. 1. In the following, we define $\theta<\pi / 2$ if the largest part of the colloids is in contact with water. $\theta$ in that

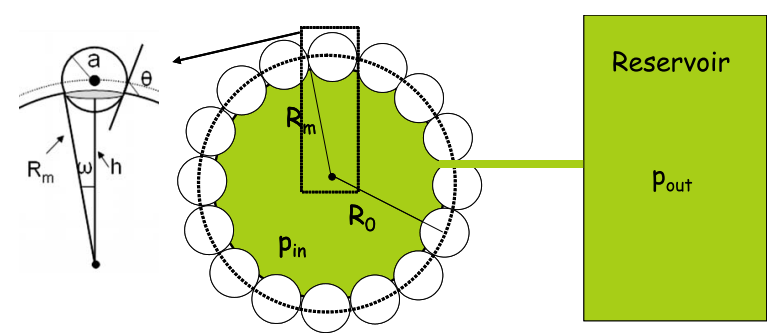

FIG. 1. (Color online) Ensemble of spherical particles adsorbed at the oil-water interface with the droplet interior connected to a reservoir with pressure $p_{\text {out }}$. In his cartoon we have $\theta<\pi / 2$ so that (see text) colloids are mainly in contact with water, and we have an $\mathrm{O} / \mathrm{W}$ (oil droplets in water) emulsion. The pressure inside the drops is $p_{i n}$. On the left the quantities that determine the interfacial areas in Eq. (1) are indicated. 

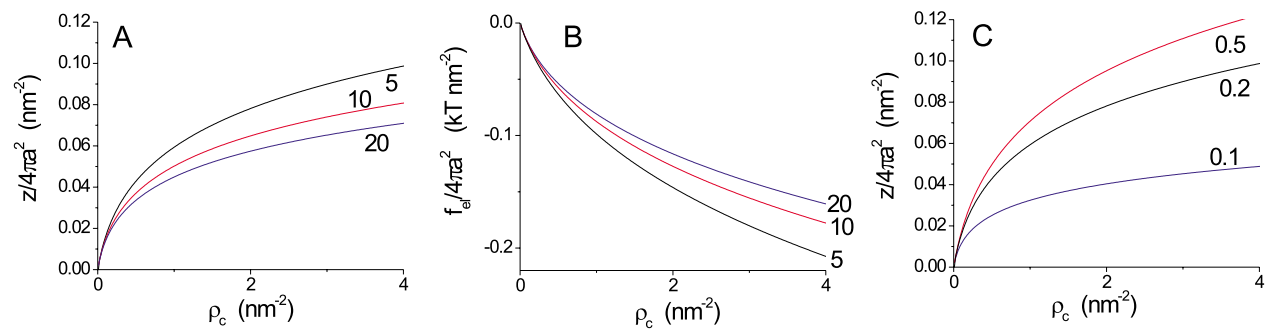

FIG. 2. (Color online) (a) Far-field or renormalized charge [Eq. (7)] per unit area as a function of the areal density of chargeable groups, $\rho_{c}$, for several values of the radius $a$ (in $\mathrm{nm}$ ) as indicated in the Figure, with $\kappa=0.2 \mathrm{~nm}^{-1}$. (b) free energy [Eq. (5)]. (c) As (a) with $a=5 \mathrm{~nm}$ and for several values of the inverse Debye length $\kappa$ as indicated in the figure. The values of $b=0.5 \mathrm{kT}$ and $\lambda_{B}=0.71 \mathrm{~nm}$.

case resembles the wetting angle of a macroscopic water drop onto a solid phase of colloidal material, both immersed in oil. We moreover define $\mathrm{O} / \mathrm{W}$ emulsions as oil drops in water, with $R_{m}>0$ and W/O as water drops in oil, with the "inverse curvature" $R_{m}<0$. Finally $f_{e l}(\theta, \omega, a)$ is the electrostatic contribution per colloid that is expected to depend upon the angles $\theta$ and $\omega$ and on the colloid radius.

In order to establish if the state where colloids are adsorbed onto spherical oil-water interfaces is indeed stable, we have to compare the (yet to be optimized) free energy Eq. (1) to the situation where there is negligible oil-water interface [11] and the colloids reside in either the water or oil phase. In other words the following condition should be fulfilled

$$
\left(\Delta f_{o}<0\right) \wedge\left(\Delta f_{w}<0\right)
$$

where the free energy difference between the emulsified state and the demixed state where all colloids reside in oil

$$
\Delta f_{o}=\frac{F}{n}-4 \pi a^{2} \gamma_{c o}
$$

The interfacial tension between colloids and water is affected by the electrostatic free energy, i.e., for a colloid fully immersed in water its interfacial tension reads $\gamma_{c w}^{(1)}=\gamma_{c w}+\frac{f_{e l}(\theta=\omega=0, a)}{4 \pi a^{2}}$ with $f_{e l}(\theta=\omega=0, a)$ the electrostatic free energy of a sphere fully immersed in water. So we write for the free energy of the emulsified state relative to the demixed state where colloids are dispersed in water

$$
\Delta f_{w}=\frac{F}{n}-4 \pi a^{2} \gamma_{c w}^{(1)}
$$

Without the electrostatic term, i.e., $f_{e l}(\theta, \omega, a)=0$ in Eq. (1), condition (2) cannot be fulfilled simultaneously and colloids will be either in water or in oil [12]. The reason is that even for an oil-water interface that is close packed with (spherical) colloids, there is at least ten percent energetically unfavorable bare oil-water interface present. The only term that potentially provides a favorable contribution to the free energy and compensates for the energy cost in creating interface comes from the electrostatic term. Such negative contributions to the free energy should not be proportional to the particle or oil-water surface areas, since this would not change Eq. (1) with $f_{e l}(\theta, \omega, a)=0$ except for a change in effective surface tension, thereby leaving the penalty for surface creation intact. However, negative contributions to $f_{e l}$ that are sublinear in the (exposed) surface areas may give rise to a favorable scenario. Imagine a particle that has a preference for oil and is barely touching the oil-water interface. In that situation the release of ions, which is entropically favorable, effectively reduces the colloid-water interfacial tension. If a larger part of the particle will be exposed to water, then Coulomb forces prevent further dissociation [13] thereby increasing the effective particle-water interfacial tension. By this mechanism deeper penetration into the water is disfavored. So by residing on the interface a particle can have optimal release of counterions while simultaneously benefitting from contact with oil. Under the appropriate conditions this double benefit may exceed the cost of the creation of bare oil-water interface. The aim of this work is to investigate this scenario.

For the electrostatic contribution in Eq. (1) we take

$$
f_{e l}=k T\left(z \ln \left(z / z_{\max }\right)+b z+c z^{2}\right) .
$$

Here $z$ is the number of charges on a colloidal particle and $z_{\max }$ is the number of dissociable or chargeable groups on a colloid. $b$ is the ionic dissociation energy being typically on the order of $k T$ in aqueous systems. The quantity $c$ in Eq. (5) takes into account the interactions between charges. For a homogeneously charged sphere we have (in the Debye Hückel approximation)

$$
c=\frac{\lambda_{B}}{2 a(1+\kappa a)}
$$

with $\lambda_{B}$ the Bjerrum length and $\kappa$ the inverse Debye length. Per construction, the value of $z$ in Eq. (5) is a far-field or renormalized value. It follows from minimizing Eq. (5) and reads as

$$
z=\frac{W\left(2 c e^{-(b+1)} z_{\max }\right)}{2 c} .
$$

Here $W(x)$ is the Lambert $\mathrm{W}$ function defined by $x=W(x) e^{W(x)} . z$ behaves as the far-field solution of the nonlinear Poisson-Boltzmann equation in the cell model pioneered by Alexander et al. in [13]: at small values of $z_{\max }$, $z \propto z_{\max }$ while at large values of $z_{\max }, z$ varies sublinearly with $z_{\max }$. The situation for colloidal particles fully immersed in aqueous solutions is illustrated in Fig. 2. The charge density on the particles of 0.05 to 0.1 charges $/ \mathrm{nm}^{2}$ corresponds to $1-2 \mu \mathrm{C} / \mathrm{cm}^{2}$. These are typical values for inorganic surfaces in water where the charge levels range between -15 and $15 \mu \mathrm{C} / \mathrm{cm}^{2}$ [14]. In case of colloidal particles partly 

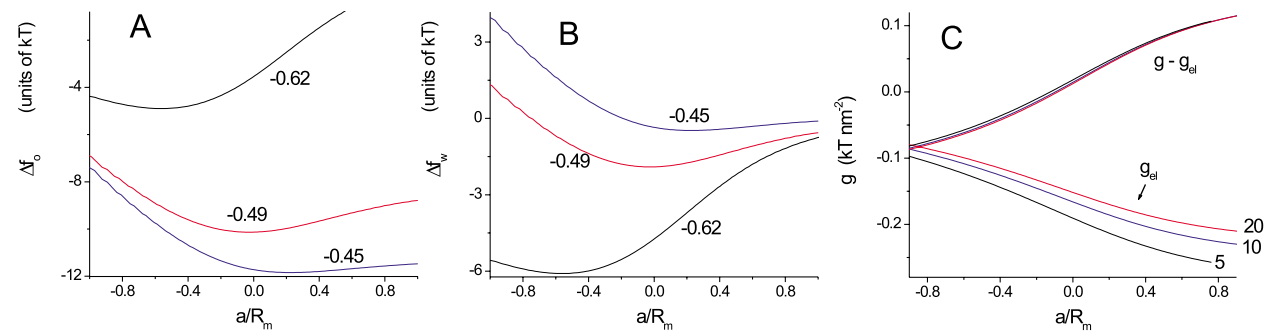

FIG. 3. (Color online) Free energies [Eq. (3)] (A), and [Eq. (4)] (B) versus curvature of the oil-water meniscus, $a / R_{m}$ for values of $\gamma_{o w}=2 \mathrm{mN} / \mathrm{m}, \rho_{c}=2 \mathrm{~nm}^{-2}, a=5 \mathrm{~nm}, b=0.5 \mathrm{kT}, \kappa=0.2 \mathrm{~nm}^{-1} . \Delta \gamma$ varies between $-0.45 \mathrm{and}-0.62 \mathrm{mN} / \mathrm{m}$ as indicated in the figure. Panel C: electrostatic part of the reduced free energy, $g_{e l}=\frac{f_{e l}}{2 \pi a^{2}}$ with $f_{e l}$ given by Eq. (5) and $g-g_{e l}=\frac{F}{2 \pi a^{2} n}-g_{e l}-\gamma_{c o}-\gamma_{c w}$ versus curvature of the oil-water meniscus for several values of the colloid radius $a$ as indicated in the figure (in $\mathrm{nm}$ ). The values of the other quantities are as in panels A and B and $T=293 \mathrm{~K}$. Note that the behavior of $g_{e l}$ reflects the trend in Fig. 2, middle.

immersed in oil, as in Fig. 1, we neglect ionic dissociation at the colloid-oil interface as charge density in oil will typically be much smaller than in water. Chargeable groups are assumed fixed and homogeneously distributed over the colloidal particle surface so that $z_{\max }=\rho_{c} A_{c w}$, with $\rho_{c}$ the areal density of chargeable groups on a colloid. We also assume that Eqs. (5) and (6) still hold for the situation depicted in Fig. 1. In other words, we neglect interactions between charges on different colloids, which seems reasonable as long as $\kappa^{-1}<a$. In order for Eq. (6) to hold, a significant fraction of the sphere should be in contact with water, which has indeed been verified, i.e., under the conditions studied here, at least a quarter of the colloid surface area is in contact with water. Moreover, $z$ should be a weakly varying function of $z_{\max }$, which indeed is the case for the high densities of chargeable groups $\rho_{c} \geq 1 \mathrm{~nm}^{-2}$ that are relevant in this work, as can be verified in Fig. 2 .

We further consider droplets where the adsorbed colloids are close-packed. This is a potentially awkward constraint. We therefore take an ensemble with fixed $R_{0}$, see Fig. 1, and fixed number of adsorbed colloids, $n$, being determined by the value of $R_{0}$. Note that close packing is a reasonable approximation for the dense packings as observed in Refs. $[5,6]$. Deviations from close packing are expected not to qualitatively change the scenario as long as the oil-water interfacial area covered by the colloids is much larger than the bare oil-water area. The maximum number of colloids that can be packed onto a spherical surface of radius $R_{0}$ is given by [15]

$$
n=\frac{2 \phi^{*}}{1-\left(1-\left(\frac{a}{R_{0}}\right)^{2}\right)^{1 / 2}},
$$

where $\phi^{*}=\frac{\pi}{2,3} \approx 0.907$ is the maximum packing fraction of disks on a flat surface [16]. We expect Eq. (8) to apply to the regime where dislocations can be neglected [17], which is the case if $a / R \geq 0.2$, see, e.g., [18]. In order to find the optimum $R_{m}$ under the constraint Eq. (8), we impose mechanical equilibrium between the drops and a macroscopic excess phase, that is, we put

$$
\Delta p=0 \quad \text { (close-packed). }
$$

Coexistence of finite size drops with excess phase, implicit in the condition Eq. (9), has indeed been observed in equi- librium Pickering emulsions [5]. Note that this condition implies an oil-water interface with zero mean curvature, an issue to be addressed in a separate publication. Despite this complication, we may assume Eq. (1) is a reasonable approximation for such an interface [19]. Next we minimize the free energy equation (1) per colloid, under the condition of constant $R_{0}$ and the number of adsorbed colloids given by Eq. (8), this condition being expressed as $R_{0}(n)$, i.e., $\left(\frac{\partial F / n}{\partial R_{m}}\right)_{R_{0}(n)}=0$. The optimal $R_{0}, R_{m}$ pairs are inserted into Eqs. (3) and (4) and the results compared to the condition Eq. (2). With reasonable values for the relevant quantities such as the areal density of chargable groups $\rho_{c}, b$, and $\gamma_{o w}$, as indicated in the caption of Fig. 3, condition (2) can be satisfied and stable emulsions are possible in this scenario. As can be seen in Fig. 3, there even is a well-defined preferred curvature as indicated by a minimum of the free energy as a function of $a / R_{m}$. In the absence of excess oil or water, emulsions are still stable with droplet radii proportional to the ratio between dispersed volume and fraction of added colloids.

In Figs. 3(A) and 3(B), optimized free-energy Eqs. (3) and (4) have been plotted as a function of curvature, $a / R_{m}$. In Fig. 3 (panel C) it can be seen that the minima arise because of competing electrostatic and non-electrostatic contributions to the free energy. Nonelectrostatic contributions tend to bend the interface in the negative (W/O) direction and reflects the empirical rule already observed by Pickering [2]; i.e., the colloidal monolayer tends to curve in the direction of the least-wetted liquid, in this case water (free energy decreases in the direction of W/O). On the other hand, the electrostatic contribution prefers positive $(\mathrm{O} / \mathrm{W})$ curvature: in that case a larger area per colloid is being exposed to water thereby increasing the degree of ionic dissociation and configurational entropy associated with that. In calculating Fig. 3 we used the experimentally observed value $\gamma_{o w} \approx 2 \mathrm{mN} / \mathrm{m}[8]$ and choose the values of $b=0.5 \mathrm{kT}$ and $\rho_{c}=2 \mathrm{~nm}^{-2}$ so that the value of the surface potential $\Psi=2 c z=-\ln \left(\frac{z}{z_{\max }} e^{1+b}\right)$ is comparable to the measured zeta potential in these systems of 1.5-2 kT, [7].

As can also be seen in Fig. 3, small variations in $\Delta \gamma=\gamma_{c o}-\gamma_{c w}$ trigger inversion from $\mathrm{W} / \mathrm{O}$ to $\mathrm{O} / \mathrm{W}$ emulsions. There is a window of stability where $-0.4 \leq \Delta \gamma / \gamma_{o w} \lesssim-0.2$ in the numerical example in Fig. 3 because of competing reference states of colloids in either oil or water. While less negative values of $\Delta \gamma$ decreases $\Delta f_{o}$, it increases $\Delta f_{w}$ so at some point, colloids will go to water. Inversion from W/O to 
$\mathrm{O} / \mathrm{W}$ emulsions can also be triggered by increasing the value of $\kappa$ (not shown).

The electrostatic part of the free energy, per unit area, increases with colloid size, as illustrated in Fig. 3(c). At the same time, the non-electrostatic contribution per unit area is more or less constant. This implies there is an upper limit on colloid size for stable emulsions, i.e., the free energy per colloid in the emulsion scales as $F / n \sim a^{2}-a^{2-\delta}$ with an effective exponent $\delta>0$. The first term is due to interfacial tensions while the second contribution is because of electrostatics. It implies electrostatics acts as a negative "line tension."

We presented a scenario for thermodynamic stability of emulsion drops covered by charged colloidal particles. Quantities that have to be plugged into the theory are close to experimental values in the experimental system in Ref. [5]. The stabilizing mechanism is ion entropy that is not available to colloids in the absence of an oil-water interface. Preference for the oil phase avoids colloids to go completely into water. The stabilizing effect depends subquadratically on colloid size, which implies an upper limit for stability. Both these effects, i.e., preference for the oil phase as well as an upper limit for the colloidal size are consistent with experiments.

Next to these conditions, additional requirements for equilibrium solid-stabilized emulsions are: (1) the value of the oil-water interfacial tension is on the order of $1 \mathrm{mN} / \mathrm{m}$; (2) the density of chargeable groups is on the order of $1 \mathrm{~nm}^{-2}$; (3) Debye length is on the order of the colloid size. We note that conditions (1) and (2) are interrelated, for example, lower values of the density of chargeable groups may still lead to spontaneous emulsification, but only if $\gamma_{o w}$ is also lowered. The physical significance of condition (3) is that if the Debye length is too large, charging up is too expensive and colloids will go to oil. If it is too small, the electrostatic contribution to $\gamma_{c w}^{(1)}$ will drive colloids to water. This opens up the possibility to switch between an emulsified and a demixed state of oil and water.

We have neglected the effect of adsorption on translational entropy of the colloids. That will contribute a few kT's and increase the values of the free energy minima in Fig. 3 without qualitatively changing the picture. A bit more worrying is that the optimal $(\mathrm{O} / \mathrm{W})$ droplet size decreases with increasing $\kappa$, which is in the opposite direction of the experiments in Ref. [5]. Thus, there are more ingredients in the experimental system in Ref. [5] than there are in the scenario reported here. A possible important effect that is not in our theory is the influence of hydrolized (and charged!) oil in the system in Ref. [5]. However, our results imply that thermodynamic stability is possible without this effect. We believe it is because of the low interfacial tension that spontaneous emulsification has only been a singular observation up till now. Even for oil-water interfaces covered with a surfactant (SDS), at low ionic strength, the interfacial tension is significantly larger than the one in Refs. [5,6], see, e.g., [20]. In principle, interfacial tension can be further lowered by adding cosurfactant(s), e.g., alcohol(s). Our work suggests that in these systems, chargeable colloids with preference for the oil phase may induce spontaneous emulsification.

We thank Stefano Sacanna, Daniela Kraft, Albert Philipse, René van Roij, Jos Zwanikken, and Martin Oettel for discussions.
[1] W. Ramsden, Proc. R. Soc. London 72, 156 (1903).

[2] S. U. Pickering, J. Chem. Soc. 91, 2001 (1907).

[3] F. Leal-Calderon and V. Schmitt, Curr. Opin. Colloid Interface Sci. 13, 217 (2008).

[4] R. Aveyard, B. P. Binks, and J. H. Clint, Adv. Colloid Interface Sci. 100-102, 503 (2003).

[5] S. Sacanna, W. K. Kegel, and A. P. Philipse, Phys. Rev. Lett. 98, 158301 (2007).

[6] S. Sacanna, W. K. Kegel, and A. P. Philipse, Langmuir 23, 10486 (2007).

[7] D. J. Kraft (to be published).

[8] S. Sacanna, Ph.D. thesis, Utrecht University, 2007.

[9] This value is lower than the one reported in [5] which was measured immediately after mixing water and TPM. The value in [8] is a constant value that is reached after several hours. The lower value is probably caused by hydrolysis of TPM that adsorbs onto the oil-water interface, thereby lowering the interfacial tension.

[10] W. K. Kegel, J. T. G. Overbeek, and H. N. W. Lekkerkerker, in Microemulsions, Fundamental and Applied Aspects, edited by P. Kumar and K. Mittal (Marcel Dekker, New York, 1999), p. 13.

[11] The pre-existing (macroscopic) oil-water interfacial area is negligible compared to the area of new oil-water-colloid interface under consideration. Moreover, in most situations the free energy to transfer colloids from oil to water; i.e., $4 \pi a^{2} \Delta \gamma$ with
$\Delta \gamma=\gamma_{c o}-\gamma_{c w}$ is under most circumstances so large that if dispersed, colloids will be either in oil or in water.

[12] In that case particles may be adsorbed onto the macroscopic oil-water interface that also exists without the presence of colloids.

[13] S. Alexander, P. M. Chaikin, P. Grant, G. J. Morales, and P. Pincus, J. Chem. Phys. 80, 5776 (1984).

[14] R. J. Hunter, Foundations of Colloid Science, 2nd ed. (Oxford University Press, Oxford, 2001).

[15] P. A. Kralchevsky, I. B. Ivanov, K. Ananthapadmanabhan, and A. Lips, Langmuir 21, 50 (2005).

[16] We find comparable results if we neglect curvature dependence of close packing and simply take $n=4 \phi^{*} \frac{R_{0}^{2}}{a^{2}}$.

[17] M. J. Bowick, D. R. Nelson, and A. Travesset, Phys. Rev. B 62, 8738 (2000).

[18] A. R. Bausch, M. J. Bowick, A. Cacciuto, A. D. Dinsmore, M. F. Hsu, D. R. Nelson, M. G. Nikolaides, A. Travesset, and D. A. Weitz, Science 299, 1716 (2003).

[19] It can be shown that in general the contact line drops as a result of the vanishing pressure with a shift proportional to $a^{2} / R_{0}$. This shift implies corrections to Eq. (1), which are of second order in curvature. These terms may shift the equilibrium size of the droplets, but are insignificant with respect to the whether the emulsification process is spontaneous or not.

[20] R. Aveyard, B. P. Binks, and J. Mead, J. Chem. Soc., Faraday Trans. 83, 2347 (1987). 